\title{
From the \#MeToo Frontlines: Incoming Interns Report a Breadth of Experiences Related to Sexual Harassment in Medical School
}

$\mathrm{J}$ Gen Intern Med 36(4):1132-3

DOI: $10.1007 / \mathrm{s} 11606-020-05873-4$

(1) Society of General Internal Medicine (This is a U.S. government work and not under copyright protection in the U.S.; foreign copyright protection may apply) 2020

\section{INTRODUCTION}

With the rise of the \#MeToo movement, sexual harassment in medicine has been placed in the spotlight, along with increasing recognition that the spectrum of sex discrimination includes microaggressions and gender bias. Recent studies have explored the prevalence of sexual harassment in medicine, finding that up to $30 \%$ of female physicians report sexual harassment in the workplace ${ }^{1}$.

Medical students are a particularly vulnerable population within medicine as they experience the widest power differentials and their ongoing grades and evaluations deepen the consequences of retaliation to reporting. The recent National Academies of Science, Engineering, and Medicine (NASEM) report places the rate of sexual harassment in medical students close to $45 \%^{2}$.

There is a significant need to decrease the high prevalence of sexual harassment in medical school with various calls for institutional changes to address the milieu in which harassment develops and thrives. However, while the prevalence of sexual harassment has been studied, there is little granularity to the data, and it is not clear what types of experiences make up the sum total reported in the literature. We believe more specific details about these experiences are important to develop targeted interventions. Therefore, we aimed to further delineate the ways in which medical students experienced sexual harassment in medicine.

\section{METHODS}

An in-person cross-sectional survey was administered to incoming interns at a large academic hospital (UPMC) about their experience with sexual harassment in medical school (IRB\#: PRO18040525). An optional question invited respondents to share experiences of sexual harassment in free-text form ("If you are willing and interested in sharing any of your

Received February 14, 2020

Accepted April 24, 2020

Published online June 8, 2020 experiences, please use the space below"). Using an inductively developed codebook and in-person adjudication, the authors examined the responses for common themes.

\section{RESULTS}

A total of 185 graduates of US medical schools returned the survey (99\% response rate), with 42 completing the optional free-text question. Of these, 33 respondents identified as female, and 9 as male. They had attended medical school in 17 different states, from all four US regions. Analysis of the responses revealed six recurring themes (see Table 1): (1) decision not to report, (2) unsuccessful reporting, (3) disproportionate "scut" work for female students, (4) inappropriate comments and touching, (5) repeat offenders with well-known reputations, and (6) male patients harassing female students. This last theme was particularly attributed to Veterans Administration (VA) patients.

\section{DISCUSSION}

More female students than male students shared negative experiences, identifying a breadth of experiences attributed to both colleagues/superiors and patients. Most importantly, many students identified the current reporting system as discouraging of reporting.

We believe that examining incoming interns to obtain information about medical school experiences optimizes accuracy. Perceived risk of retaliation is diminished in this population comprised of students who have already matched and graduated, most of whom have even left the institution being queried. Additionally, we believe the study sample represents a relatively representative sample as the participants attended a large range of institutions prior to joining their post-graduate training institution.

Recall bias is a limitation of our study, which we attempted to minimize by collecting data in early July, within weeks of graduation from medical school.

Given the variety of experiences shared by incoming trainees, tackling sexual harassment must include targeted approaches for each issue. The current reporting system appears to be insufficient and reforms are needed to develop an improved system that encourages and supports trainees to report inappropriate experiences. 
Table 1 Thematic Analysis of Sexual Harassment Experiences Reported by Incoming Interns

\begin{tabular}{l}
\hline Theme \\
Male patients harassing \\
female students \\
Inappropriate comments and \\
touching
\end{tabular}

Repeat offenders with wellknown reputation

Disproportionate "scut" work for female students

Decision not to report

Unsuccessful reporting
Representative quotation(s)

"... a patient at the VA hospital who told me during the physical exam that if I leaned over him one more time he was going to 'grab my tits"'

"Older male patients...getting in my physical space, using sexual terms to make me uncomfortable, or asking to see me later outside the hospital" "A male resident...touched $\boldsymbol{m y}$ stomach and my shoulders. It made me really uncomfortable"

"I had an attending joke about looking down my shirt in the OR." "...I had been warned about this preceptor by other students and was told we still used him because he did a lot of teaching"

"The attending was known to 'put down' women...told one of my female co-workers 'you should wake up an hour earlier to blow dry your hair \& put on make-up"

"A male med student and I were working with the same resident. He repeatedly asked the male medical student to help with patient care, and asked me to help with non-clinical menial tasks."

"[An attending] would make me call patients to see if they were coming in to the appointments while he let my [male] coworker see patients."

"I had several experiences with other female medical students discussing whether or not to report harassment. Usually we all decided together not to report due to fear of repercussions." "I...witnessed intense sexual harassment of female residents by attendings and was told by residents not to report it."

"Apparently he'd been reported formally before and nothing had changed. Nothing came of my report."
Emmanuelle Yecies, MD, $\mathrm{MS}^{1,2}$

Melissa McNeil, MD, MPH ${ }^{1,2}$

${ }^{1}$ Division of General Internal Medicine, University of Pittsburgh School of Medicine,

Pittsburgh, PA, USA

${ }^{2}$ Department of Medicine, VA Pittsburgh Healthcare System,

Pittsburgh, PA, USA

Corresponding Author: Emmanuelle Yecies, MD, MS; Division of General Internal Medicine, University of Pittsburgh School of Medicine Pittsburgh, PA, USA (e-mail: Yeciese@upmc.edu).

\section{Compliance with Ethical Standards:}

Conflict of Interest: The authors declare that they do not have a conflict of interest.

\section{REFERENCES}

1. Jagsi R, Griffith KA, Jones R, Perumalswami CR, Ubel P, Stewart A Sexual Harassment and Discrimination Experiences of Academic Medical Faculty. JAMA. 2016;315(19):2120-1. PMID: 27187307

2. National Academies of Sciences, Engineering, and Medicine. Sexual Harassment of Women: Climate, Culture, and Consequences in Academic Sciences, Engineering, and Medicine. Washington, DC: The National Academies Press; 2018. 